\title{
Microtubule Electrical Oscillations and Hippocampal Function
}

\author{
María del Rocío Cantero, Horacio F. Cantiello
}

Laboratorio de Canales lónicos, Instituto Multidisciplinario de Salud, Tecnología y Desarrollo (IMSaTeD, CONICET-UNSE) Santiago del Estero, Argentino

\section{Article Info}

\section{Article Notes}

Received: May 22, 2020

Accepted: June 23, 2020

\section{${ }^{*}$ Correspondence:}

Dr. Horacio F. Cantiello. IMSaTeD (CONICET-UNSE),

Laboratorios Centrales. Ruta Nacional N9 km 1125 S/N, Villa

El Zanjón, Santiago del Estero, Argentina;

E-mail: hcantiello@yahoo.com.ar.

(c) 2020 Cantiello HF. This article is distributed under the terms of the Creative Commons Attribution 4.0 International License

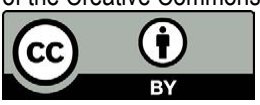

\section{Keywords :}

Microtubules

Electrical Oscillations

Hippocampal

Brain

Neurons

\section{ABSTRACT}

Microtubules (MTs) are long cylindrical structures of the cytoskeleton that control cell division, vesicular transport, and the shape of cells. MTs are highly charged and behave as nonlinear electrical transmission lines. However, comparatively little is known about the role(s) these nonlinear electrical properties of MTs play in cell function. MTs form bundles, which are particularly prominent in neurons, where they help developmentally define axons and dendrites. The present review summarizes recent work from our laboratory, which demonstrated that 1) bundles of rat brain MTs spontaneously generate electrical oscillations and bursts of electrical activity similar to action potentials; 2) actin filaments control electrostatically the oscillatory response of brain MTs; and 3) neurites of cultured mouse hippocampal neurons generate and propagate electrical oscillations thus, providing a cellular correlate to the isolated MT oscillations. Electrical oscillations are an intrinsic property of brain MT bundles, which may have important implications in the control of various neuronal functions, including a contribution to the intrinsic oscillatory modes of neurons, and thus to higher brain functions, including the formation of memory and the onset of consciousness.

\section{Electrical Oscillations of Bundles of Brain Microtubules}

MTs are unique components of the cytoskeleton that form a wide variety of intracellular superstructures ${ }^{1}$. Highly polarized cells such as neurons present two distinct cellular domains, namely the axon and multiple shorter dendrites that either transmit or receive electrical signals, respectively. In both axons and dendrites, MTs form dense parallel arrays known as bundles, which are required for neuronal growth and maintenance of neurites ${ }^{2}$. MTs are formed of highly charged $\alpha, \beta$ tubulin heterodimeric units that behave as biological transistors supporting, amplifying and axially propagating electrical signals ${ }^{3}$. Within the cytoplasm MT-generated variable currents may contribute to the presence and modulation of large intracellular electric fields, which in turn, will help control cell function.

To determine the electrical activity of bundles of rat brain MTs, we recently used the loose patch-clamp configuration technique ${ }^{4}$ on MT bundles isolated from rat brain. These MT structures displayed spontaneous electrical activity consistent with self-sustained oscillations that responded directly to the magnitude of the electrical stimulus (Figure 1). Most frequently, the initial response consisted of strong bursts of oscillations that varied both in amplitude and frequency in the absence of any change in driving force. Interestingly, mean currents were often linear respect to voltage, although spontaneous changes in amplitude of the cyclic regimes were observed as well. Although similar, the oscillatory response of the bundles was richer than that reported for brain MT sheets ${ }^{5}$ and more coherent than that observed in isolated $\mathrm{MTs}^{6}$. Thus, the geometry of the MT assembly 

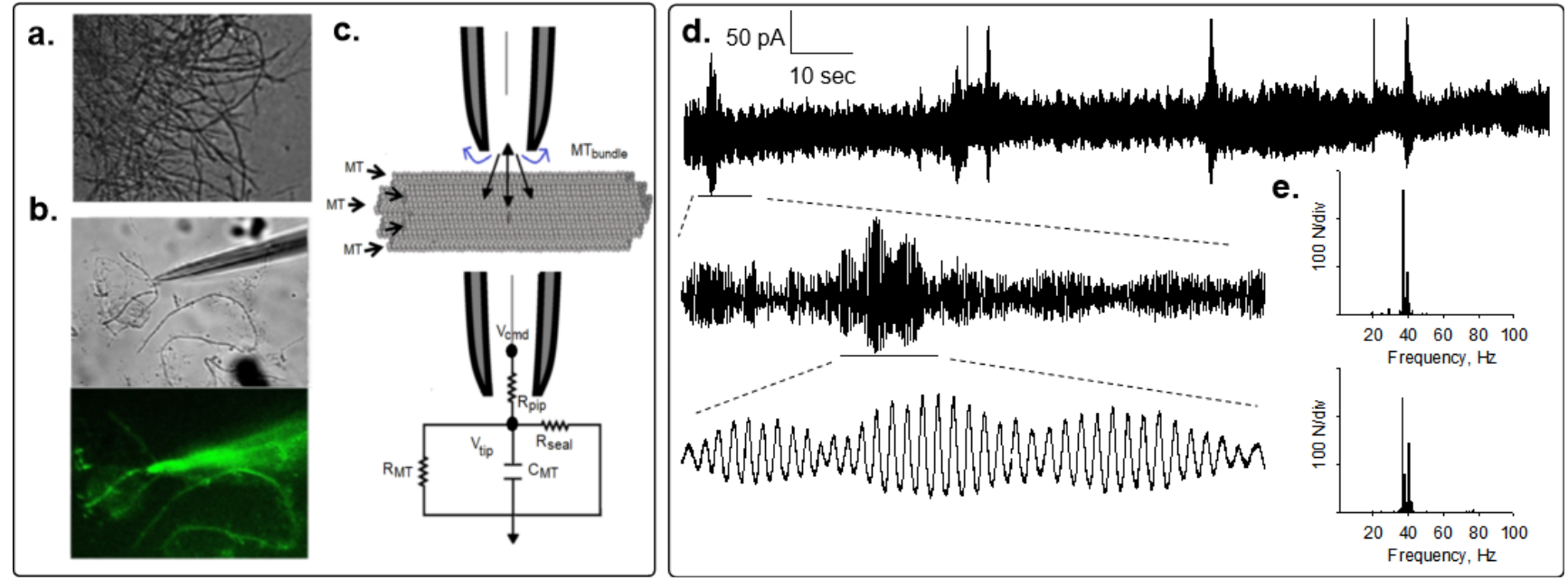

Figure 1: Electrical oscillations of brain MT bundles. Rat brain MT bundles were obtained as reported ${ }^{4}$. a. DIC image of a mesh of MT bundles. b. A patch pipette approaching an MT bundle. Bottom image shows live immunochemical MT labeling by addition of anti- $\alpha$ tubulin antibody to the preparation. c. Loose patch configuration showing the MT bundle and the patch pipette and schematics of the "loose-patch" clamp configuration to obtain electrical signals of MT bundles. d. Spontaneous changes in oscillatory behavior of patched MT bundle showing the various spontaneous regimes without any change in driving force. Periodic changes in the amplitude of the oscillations showed fractal "envelopes" of increasingly lower frequency and different oscillatory regimes. e. Power spectra for expanded tracings in (d.), where the $39 \mathrm{~Hz}$ fundamental frequency is present.

may be an important factor in the nonlinear electrical outcome. However, the fundamental frequency of $39 \mathrm{~Hz}$ in the MT bundles prevailed, also showing changes in regime and sudden death ${ }^{4}$. MT bundles also elicited highly synchronized trains of current oscillations that mimicked bursts of action potentials. Higher and lower fundamental frequencies were also observed, regardless of the applied holding potential. The MT stabilizer paclitaxel (Taxol, 10 $\mu \mathrm{M}$ ) known to traverse the nanopores at the MT surface, inhibited the brain MT bundle oscillations ${ }^{4}$.

\section{Regulation by Actin of Brain Microtubule Electrical Oscillations}

Actin filaments and MTs interact with each other for a range of dynamic cellular processes, including intrinsic structural support in the formation of axons to send, and dendritic networks to receive synaptic signals, respectively ${ }^{7}$. Actin filaments display a number of nonlinear electrical properties of their own, including a nonlinear electro-osmotic behavior ${ }^{8}$, and the ability to conduct ion condensation waves ${ }^{9}$. Actin filaments behave as biological cables. We recently evaluated the effect of actin polymerization on the electrical oscillations of brain $\mathrm{MTs}^{10}$. Electrical signals were collected from high-seal brain MT sheets. Addition of a polymerizing concentration of monomeric (G)-actin increased the amplitude of spontaneous oscillations from MT sheets after a 2-min lag time, which is consistent with the nucleation process of actin ${ }^{11}$. Larger transferred charge was observed without any changes in the oscillatory frequency. The high-affinity G-actin binding protein deoxyribonuclease I (DNAse I) that prevents actin polymerization ${ }^{12}$ was instead inhibitory in the presence but not absence of G-actin, confirming the effect of actin polymerization in the regulation of the oscillations. Compared to G-actin, addition of prepolymerized (F)-actin produced a faster and higher stimulatory effect on the magnitude of the MT sheet oscillations, but preserving the fundamental oscillatory frequency at $\sim 38 \mathrm{~Hz}$. The regulatory role of F-actin in the brain MT sheet oscillations could be mediated by proteins capable of binding both actin and tubulin, including MAP2, MAP2c and $\mathrm{Tau}^{13}$. This interaction between F-actin and MTs could also be electrostatic, because actin polymerizes on charged surfaces ${ }^{14}$. An F-actin-MT interaction may be essential in the gating of cytoskeleton-associated ion channels in such neuronal compartments as the dendritic spine.

\section{Cytoskeleton Oscillations and the Hippocampus}

It has long been speculated that the brain stores memories by altering the strength of large assemblies of interconnected neurons ${ }^{15}$. At the cellular-molecular level this approximation leads to synaptic plasticity mechanisms that constitute the physical correlates of memory storage ${ }^{16}$. Long-term potentiation (LTP) in neurons ${ }^{17}$ is widely believed to be associated with the memories stored in the brain ${ }^{18}$. LTP was initially discovered in the hippocampus ${ }^{17}$, a part of the limbic system associated with response inhibition, the formation of new memories, and also spatial cognition ${ }^{19}$. Surgical destruction of the hippocampi in humans produces severe anterograde and partial retrograde amnesia, with loss of the ability to form new episodic memories ${ }^{20}$. 
An interesting aspect of mammalian hippocampi (and other parts of the brain), including humans, is the presence of endogenous electrical oscillations, particularly slow waves known as theta-alpha frequencies ${ }^{21}$. In the rat, these large 6-9 $\mathrm{Hz}$ frequency waves appear during both alert behavior and REM (dreaming) sleep ${ }^{22}$. The hippocampus generates some of the largest EEG signals as theta waves ${ }^{23}$ that reflect sub-threshold membrane potentials that strongly modulate the spiking of hippocampal neurons and synchronize the hippocampus in travelling wave patterns ${ }^{24}$. Theta waves have been linked to mnemonic processes ${ }^{25}$. Although the molecular mechanisms that generate electrical oscillations in hippocampal neurons have yet to be firmly disclosed, the intrinsic oscillatory properties of these cells ${ }^{26}$, have been associated with identifiable ionic conductances including GABAA and NMDA receptors ${ }^{27,28}$, dendritic $\mathrm{Ca}^{2+}$ currents that amplify NMDA receptor-activated somatic oscillations, and include $\mathrm{R}$-, and several $\mathrm{L}-$ and $\mathrm{N}$-type $\mathrm{Ca}^{2+}$ channels. The hippocampal EEG of animals also show short-lived memory-associated high-frequency EEG oscillations called sharp waves and "ripples" in the $150-200 \mathrm{~Hz}^{29}$.

To explore whether the neuronal cytoskeleton participates in the genesis of electrical oscillatory patterns, we cultured adult mouse hippocampal neurons ${ }^{4}$. After several days in culture and the development of connecting neurites, we permeabilized the cells with Triton-X to access the cytoskeleton (Figure 2), and applied the double-patch clamp method, as reported ${ }^{3}$. Upon voltage clamping, low frequency electrical oscillations were observed at both ends of the neurite, which displayed different frequencies and complex behaviors, depending on the polarity of the bias. Mirror images at both ends indicated the propagation of the electrical oscillations along the length of the cytoskeleton. Interestingly, the electrical oscillations observed in the hippocampal neurites when one end (stimulus site) was voltage-stimulated, was mirrored at the other end of the neurite (collection site), thus showing propagation in a manner rather similar to that originally found in isolated $\mathrm{MTs}^{3}$. However, correlation of signals observed at both sides suggested much higher propagation velocity than in isolated MTs. Low frequencies with fundamental peaks similar to those observed in isolated neurons were also identified.

\section{An Integrated Information Processing Model of the Neuron}

The current paradigm for function of the nervous system relates to both excitatory and inhibitory interactions between neurons and also to their "intrinsic electrical activity"26. Although this paradigm includes both passive and active membrane characteristics, it does not include the neuronal cytoskeleton. Several specialized structures, including dendritic spines and growth cones, are enriched with highly dynamic actin filament networks, which are essential to experience-related plasticity, and changes associated with neural stimulation ${ }^{30}$. Morphological changes in actin-rich dendritic spines correlate with LTP in hippocampal tissue slices of behaving animals ${ }^{31}$. However, the capacity of sustaining and propagating electrical signals ${ }^{9}$ as ionic waves, makes actin filaments behave like "cables" that act as transmission lines. MTs in turn, generate, propagate and amplify electrical oscillations, such that they behave as active antennae ${ }^{3,4}$. In this context, we hypothesize that the neuronal cytoskeleton may bring forth a new capacity to handle and encode electrical information at a subcellular level. Cytoskeletal structures could be thought to as contributing to such events as reported in "memory consolidation", where short-term memories convert into long-term memories ${ }^{32}$. The
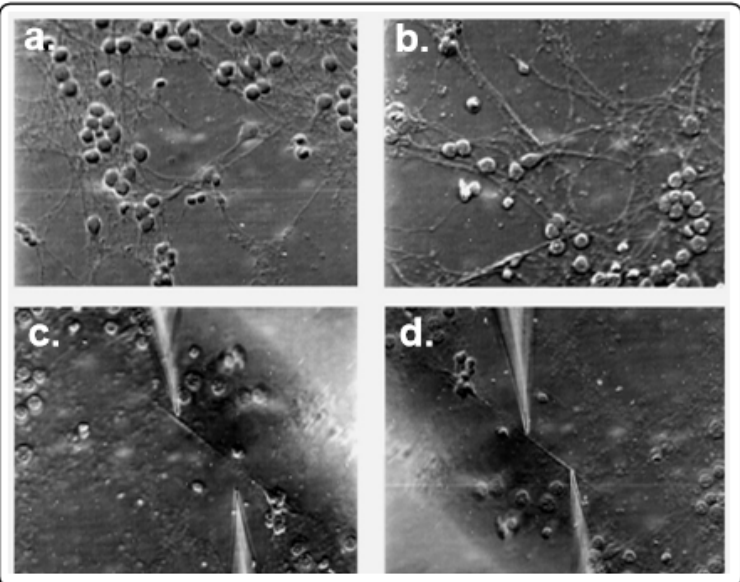
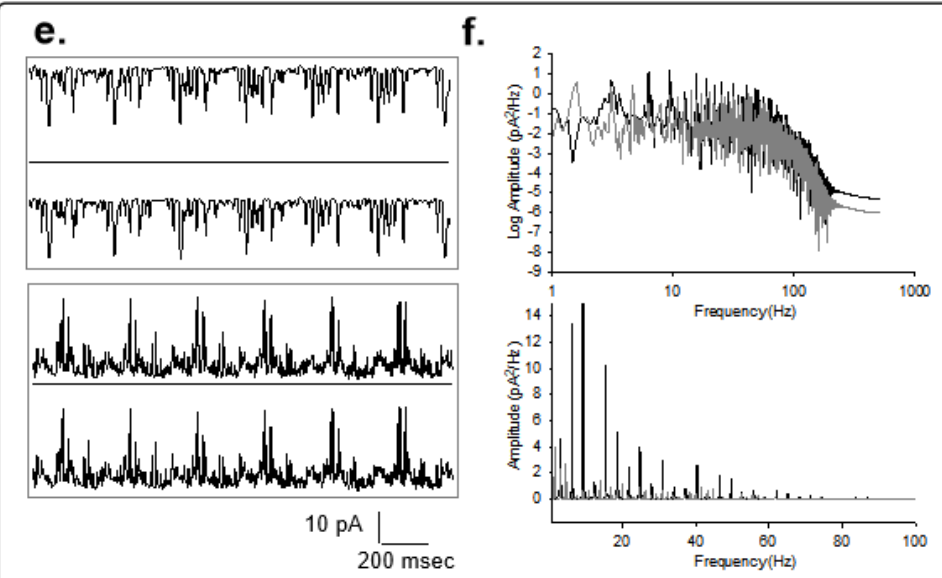

Figure 2: Electrical oscillations of hippocampal neurites. a,b. Images of one-week old cultured hippocampal neurons(x20), before and after cell membrane permeabilization by addition of $1 \%(v / v)$ Triton- $X$ to the bathing solution. $\mathbf{c}, \mathbf{d}$ Permeabilized neurites before and after double patching with patch-clamp pipettes as shown in the actual locations of electrical recording. e. Spontaneous electrical oscillations observed in one pipette and mirror images collected on the second pipette. f. Power spectra of four times the length of the tracings shown on (e), for the positive (Black) and negative (Gray) biased signals, respectively. Linear-Linear plots for respective spectra are also shown (Bottom). 
electrical activity of the neuronal cytoskeleton would also modulate membrane imbedded ion channel activity. The cytoskeleton-regulated temporal behavior of ion channel function may lead to changes in synaptic strengthening, LTP, and memory enhancement.

Our studies indicate that the electrical oscillations of brain MTs, may contribute to the various oscillatory regimes of individual neurons. The same as with MT oscillations, neural oscillations are governed by the resting potential of the cell, such that hyperpolarized cells will fire at higher frequencies near $10 \mathrm{~Hz}^{33}$. Thalamic neurons have, in addition to the low frequency rhythms, a gamma rhythm ${ }^{34}$, in the frequency range observed in isolated brain MTs $(\sim 39-40 \mathrm{~Hz})$. This is particularly appealing because the dendrite-generated gamma oscillations ${ }^{35}$ are essential in the generation of brain gamma band activity and cognitive functions ${ }^{36}$. Forty $\mathrm{Hz}$ oscillations are observed in the cortex during physiological stimulation of the visual ${ }^{37}$ or auditory corte ${ }^{38}$ during attention states in animals, and the execution of complicated tasks in humans ${ }^{39}$. Similar activity as measured by electric and magnetic means in humans correlates with cognitive tasks ${ }^{40}$.

\section{Concluding Remarks and Perspective}

In summary, our recent studies determined that bundles of brain MTs are electrically active, generating electrical oscillations in the $39 \mathrm{~Hz}$ range that correlate well with oscillatory activity observed in neurons and brain function. It is envisioned that to regulate and condition specific ionic conductances, actin networks may transmit MT-generated electrical oscillations to ion channel populated membrane locations such as dendritic spines. Electrical oscillations of MTs could be implicated in the electric fields in the brain, to help address open questions of higher brain functions, including the issues of anesthesia ${ }^{41}$, and consciousness ${ }^{42}$. Electrical oscillations by MT bundles open a novel field of biological signaling, particularly in neuron function.

\section{Funding Information}

Original studies of our laboratory were funded by Agencia-MINCyT FONCyT PICT 2016 N³739

\section{Conflict of Interest Statement}

The authors declare that there is no conflict of interest regarding the publication of this article

\section{References}

1. Desai A, Mitchison TJ. Microtubule polymerization dynamics. Annu Rev Cell Dev Biol. 1997; 13: 83-117.

2. Conde $\mathrm{C}$, Cáceres $\mathrm{A}$. Microtubule assembly, organization and dynamics in axons and dendrites. Nature Rev Neurosci. 2009; 10: 319-332.

3. Priel A, Ramos AJ, Tuszynski JA, et al. A biopolymer transistor: electrical amplification by microtubules. Biophys J. 2006; 90: 46394643.

4. Cantero MR, Villa Etchegoyen C, Perez PL, et al. Bundles of brain microtubules generate electrical oscillations. Sci Rep. 2018; 8(1): 11899.

5. Cantero MR, Perez PL, Smoler M, et al. Electrical oscillations in twodimensional microtubular structures. Sci Rep. 2016; 6: 27143.

6. Gutierrez BC, Cantiello HF, Cantero MR. Electrical oscillations of brain microtubules. bioRxiv. 2020; 2020. 04.21.054155.

7. Stiess M, Bradke F. Neuronal polarization: the cytoskeleton leads the way. Developmental Neurobiology. 2011; 71: 430-444.

8. Cantiello HF, Patenaude C, Zaner K. Osmotically induced electrical signals from actin filaments. Biophys J. 1991; 59: 1284-1289.

9. Lin EC, Cantiello HF. A novel method to study the electrodynamic behavior of actin filaments. Evidence for cable-like properties of actin. Biophys J. 1993; 65: 1371-1378.

10. Cantero MR, Gutierrez BC, Cantiello HF. Actin filaments modúlate electrical activity of brain microtubule protein two-dimensional sheets. Cytoskeleton (Hoboken). 2020; 77(3-4): 167-177.

11. Amos LA. Microtubule structure and its stabilisation. Org Biomol Chem. 2004; 2: 2153-2160.

12. Hitchcock SE, Carlsson L, Lindberg U. Depolymerization of F-actin by deoxyrybonuclease I. Cell. 1976; 7: 531-542.

13. Correas I, Padilla R, Ávila J. The tubulin-binding sequence of brain microtubule-associated proteins, tau and MAP-2, is also involved in actin binding. Biochem J. 1990; 269: 61-64.

14. Renault A, Lenne PF, Zakri C, et al. Surface-induced polymerization of actin. Biophys J. 1999; 76: 1580-1590.

15. Nadel L, O'Keefe J, Black A. Slam on the brakes: a critique of Altman, Brunner, and Bayer's response-inhibition model of hippocampal function. Behav Biol. 1975; 14(2): 151-162.

16. Kandel EF, Squire LF. Neuroscience: breaking down scientific barriers to the study of brain and mind. Science. 2000; 290: 1113-1120.

17. Lømo T. Frequency potentiation of excitatory synaptic activity in the dentate area of the hippocampal formation. Acta Physiol Scand. 1966; 68(277): 128.

18. Malenka RC, Bear MF. LTP and LTD: an embarrassment of riches Neuron. 2004; 44 (1): 5-21.

19. Squire LR. Memory and the hippocampus: a synthesis from findings with rats, monkeys, and humans. Psychol Rev. 1992; 99(2): 195-231.

20. Squire LR. The legacy of patient H.M. for neuroscience. Neuron. 2009; $61(1): 6-9$

21. Arnolds DE, Lopes da Silva FH, Aitink JW, et al. The spectral properties of hippocampal EEG related to behaviour in man. Electroencephalogr Clin Neurophysiol. 1980; 50: 324-328.

22. Buzsáki G, Chen LS, Gage FH. Chapter 19: Spatial organization of physiological activity in the hippocampal region: Relevance to memory formation. Prog Brain Res. 1990; 83: 257-268.

23. Buzsáki G. Theta oscillations in the hippocampus. Neuron. 2002; 33(3): 325-240.

24. Lubenov EV, Siapas AG. Hippocampal theta oscillations are travelling waves. Nature. 2009; 459 (7246): 534-539.

25. Miller R. Cortico-hippocampal interplay: self-organizing phase-locked loops for indexing memories. Psychobiology. 1989; 17: 115-128.

26. Llinás RR. The intrinsic electrophysiological properties of mammalian neurons: insights into central nervous system function. Science. 1988; 242: 1654-1664.

27. Kramis R, Vanderwolf $\mathrm{CH}$, Bland $\mathrm{BH}$. Two types of hippocampal rhythmical slow activity in both the rabbit and the rat: relations to behavior and effects of atropine, diethyl ether, urethane, and pentobarbital. Exp Neurol. 1975; 49: 58-85. 
28. Soltesz I, Deschénes M. Low- and high-frequency membrane potential oscillations during theta activity in CA1 and CA3 pyramidal neurons of the rat hippocampus under ketamine-xylazine anesthesia. J Neurophysiol. 1993; 70: 97-116.

29. Buzsáki G. Hippocampal sharp waves: their origin and significance. Brain Res. 1986; 398(2): 242-252.

30. Honkura N, Matsuzaki M, Noguchi J, et al. The subspine organization of actin fibers regulates the structure and plasticity of dendritic spines. Neuron. 2008; 57(5): 719-729.

31. Yuste R, Bonhoeffer T. Morphological changes in dendritic spines associated with long-term synaptic plasticity. Annu Rev Neurosci. 2001; 24: 1071-1089.

32. Nelson TJ, Backlund Jr PS, Alkon DL. Hippocampal protein-protein interactions in spatial memory. Hippocampus. 2004; 14(1): 46-57.

33. Makarenko V, Llinás R. Experimentally determined chaotic phase synchronization in a neuronal system. Proc Natl Acad Sci USA. 1998; 95: 15747-15752.

34. Deschenes M, Paradis M, Roy JP, et al. Electrophysiology of neurons of lateral thalamic nuclei in cat, resting properties and burst discharges. J Neurophysiol. 1984; 51: 1196-1219.

35. Pedroarena C, Llinás R. Dendritic calcium conductances generate high frequency oscillation in thalamocortical neurons. Proc Natl Acad Sci USA. 1997; 94: 724-728.
36. Llinás R, Choi S, Urbano FJ, et al. Gamma band deficiency and abnormal thalamocortical activity in P/Q-type channel mutant mice. Proc Natl Acad Sci USA. 2007; 104: 17819-17824.

37. Gray CM, Konig P, Engel AK, et al. Oscillatory responses in cat visual cortex exhibit inter-columnar synchronization which reflects global stimulus properties. Nature. 1989; 338: 334-337.

38. Johnson BW, Weinberg H, Ribary U, et al. Topographic distribution of the $40-\mathrm{Hz}$ auditory evoked-related potential in normal and aged subjects. Brain Topogr. 1988; 1: 117-124.

39. Sheer D. Focused arousal, 40-Hz EEG and dysfunction. In Self Regulation of the Brain and Behavior, eds TH Elbert, B Rockstroh, W Lutzenberger, N Birbaumer (Berlin: Springer-Verlag). 1984; 6484.

40. Ribary U, Ioannides AA, Singh KD, et al. Magnetic field tomography (MFT) of coherent thalamo-cortical 40-Hz oscillation in humans. Proc Natl Acad Sci USA. 1991; 88: 11037-11041.

41. Emerson DJ, Weiser BP, Psonis J, et al. Direct modulation of microtubule stability contributes to anthracene general anesthesia. Am Chem Soc. 2012; 135(14); 5389-5398.

42. Hameroff S, Penrose R. Orchestrated reduction of quantum coherence in brain microtubules: A model for consciousness. Math Comp Simul. 1996; 40: 453-480. 